Article

\title{
Integrated Phenotypic-Genotypic Analysis of Latilactobacillus sakei from Different Niches
}

\author{
Ying Chen ${ }^{1}$, Nan Li ${ }^{2}$, Shenxi Zhao', Chuan Zhang1, Nanzhen Qiao', Hui Duan', Yue Xiao', Bowen Yan ${ }^{1,3}$, Jianxin \\ Zhao ${ }^{1,3}$, Fengwei Tian ${ }^{1,3}$, Qixiao Zhai ${ }^{1,3}$, Leilei Yu ${ }^{1,3^{*}}$, Wei Chen ${ }^{1,3}$ \\ 1 State Key Laboratory of Food Science and Technology, School of Food Science and Technology, Jiangnan \\ University, Wuxi 214122, People's Republic of China \\ 2 State Key Laboratory of Dairy Biotechnology, Shanghai Engineering Research Center of Dairy Biotechnol- \\ ogy, Dairy Research Institute, Bright Dairy \& Food Co., Ltd., Shanghai 200436, China \\ 3 National Engineering Research Center for Functional Food, Jiangnan University, Wuxi, Jiangsu 214122, \\ China \\ 4 University of Alberta, Department of Agricultural, Food and Nutritional Science, Edmonton, Canada \\ * Correspondence: Dr. Leilei Yu; Tel: 86-510-85912155; Fax: 86-510-8591215; E-mail: edyulei@126.com
}

\begin{abstract}
Increasing attention has been paid to the potential probiotic effects of Latilactobacillus sakei. To explore the genetic diversity of L. sakei, 14 strains isolated from different niches (feces, fermented kimchi and meat products) and 54 published strains were compared and analyzed. The results showed that the average genome size and GC content of $L$. sakei were $1.98 \mathrm{Mb}$ and $41.22 \%$, respectively. Its core genome mainly encodes translation and transcription, amino acid synthesis, glucose metabolism and defense functions. L. sakei has an open pan-genomic characteristics, and its pangene curve shows an upward trend. L. sakei has open pan-genome feature, and its pan-genome curve is on the rise. The genetic diversity of L. sakei is mainly reflected in carbohydrate utilization, antibiotic tolerance, and immune/competition-related factors, such as clustering regular interval short palindromic repeat sequence (CRISPR)-Cas. The CRISPR system is mainly IIA type, and a few are IIC types. This work provides a basis for the study of this species.
\end{abstract}

Keywords: Latilactobacillus sakei; comparative genomics; carbohydrate utilization; antibiotic tolerance; CRISPR-Cas

\section{Introduction}

Latilactobacillus sakei is a ubiquitous psychrophilic lactobacillus, which was first isolated from sake by KATAGIR et al. [1] in 1934. This species is a gram-positive bacterium, that exists in many niches, such as sour dough [2], fermented vegetables [3], fermented meat products [4] and human feces [5]. L. sakei has attracted the attention of researchers owing to its prominent bacteriocin-producing ability. The species can produce a various class II bacteriocins such as Sakacin Q [6] and Sakacin P [7] and can inhibit a variety of food-borne pathogenic bacteria such as Listeria monocytogenes [8] and Staphylococcus aureus [9], which also promote its application in food industry. Gao et al. [10] found that adding L. sakei $\mathrm{C} 2$ and its bacteriocin can inhibit pathogenic bacteria and prevent lipid oxidation of vacuum-packed cooked ham slices at cold storage temperatures. In addition, L. sakei is used as a starter for fermented meat products [11] and kimchi [12] in the food industry, ensuring product quality and accelerates the fermentation rate. It is worth noting that $L$. sakei also has a variety of probiotic functions, such as regulating immunity [13], improving metabolic syndrome [14] and relieving inflammatory diseases [15]. In recent years, some studies have shown that $L$. sakei can reduce the expression of pro-inflammatory cytokines related to psoriasis and improve the severity of psoriasis in mice [16]. Other research results show that L. sakei K040706 can enhance the phagocytosis of macrophages and increase the expression of immune regulators such as inducible nitric oxide synthase (iNOS) and cytokines, which can be regarded as a candidate drug for immune stimulation [17]. 
In 2005, Chaillou et al. [18] sequenced the genome of L. sakei $23 \mathrm{~K}$ for the first time, which gives a foundation for the development of genome sequencing of L. sakei. With genome technology development, the genetic diversity and functional diversity of bacteria have been extensively studied. Chaillou et al. [19] revealed that L. sakei has three different phylogenetic lineages influenced by its different habitats. Nyquist et al. [20] analyzed the genome of L. sakei isolated from processed meat products, and found that the genes involved in nucleoside elimination, arginine catabolism and coping with redox and oxygen level changes of these strains were all retained, which may be the key factors for their survival in meat products. Studies have shown that niches affect the genetic characteristics and evolution direction of specific species, and these strains show specific host adaptability in the evolution process. However, at present, the genome analysis of L. sakei is limited to a few isolated sources such as fermented meat products, and the gene analysis of $L$. sakei from feces and pickles is limited. To further explore the genetic information and evolution of L. sakei, more genomic analysis is necessary.

In this study, 14 L. sakei strains were isolated, from different niches, such as feces and fermented foods. In addition, we downloaded the published genome information of $54 \mathrm{~L}$. sakei strains from National Center for Biotechnology Information (NCBI), and analyzed the genetic diversity of the core genome, pan-genome, carbohydrate utilization enzyme and CRISPR-Cas system of $68 \mathrm{~L}$. sakei strains using bioinformatics.

\section{Materials and Methods}

\subsection{Isolation of strains, genome sequencing and data assembly}

A total of 14 L. sakei strains were isolated from fecal samples, fermented meat products and fermented vegetable products in different regions of China, as shown in Table 1. The strains were cultured in De Man, Rogosa and Sharpe (MRS) medium [21] and incubated for $24 \mathrm{~h}$ at $37^{\circ} \mathrm{C}$. All the identified L. sakei strains using $16 \mathrm{~S}$ rRNA sequencing were stored at $-80^{\circ} \mathrm{C}$ in $30 \%$ glycerol. Illumina Hiseq $\times 10$ platform (Majorbio BioTech Co, Shanghai, China) was used to sequence the draft genomes of L. sakei, and 2×150 bp paired-end libraries and pairedend library with an average read length of about $400 \mathrm{bp}$ were constructed. SOAPde-novo is used to assemble the reads, and GapCloser software was used to fill the local inner gaps [22]. In addition, 54 publicly available genomes of L. sakei from NCBI (https://www.ncbi.nlm.nih.gov/) were used in this study.

Table 1. The information of 68 Latilactobacillus sakei strains.

\begin{tabular}{cccccc}
\hline Strain & Source & Genome size $(\mathrm{Mb})$ & GC $(\%)$ & CDS no. & Reference \\
\hline FAHWH17-L1 & Human feces & 1.90 & 41.82 & 1860 & This work \\
FJLHD1M1 & Human feces & 1.98 & 41.81 & 1969 & This work \\
FJLHD26M6 & Human feces & 1.98 & 41.79 & 1950 & This work \\
FXJWS8M1 & Human feces & 2.11 & 41.00 & 2142 & This work \\
FZJHZ2M8 & Human feces & 2.18 & 41.61 & 2085 & This work \\
GD179 & Human feces & 1.88 & 41.83 & 1856 & This work \\
FLEC01 & Human feces & 1.96 & 41.24 & 1919 & {$[23]$} \\
QYNXSBNJH59L1 & Sour cowhide & 1.94 & 41.77 & 1928 & This work \\
QJSSZ1L4 & Air-dried & 1.90 & 41.92 & 1876 & This work \\
& sausage & & & & This work
\end{tabular}




\begin{tabular}{|c|c|c|c|c|c|}
\hline DSM15831 & Meat & 1.99 & 41.00 & 1924 & [24] \\
\hline RI-496 & Meat & 2.02 & 40.90 & 1934 & [24] \\
\hline RI-499 & Meat & 1.91 & 41.00 & 1838 & [24] \\
\hline RI-500 & Meat & 2.03 & 40.90 & 1992 & [24] \\
\hline RI-394 & Meat & 1.94 & 41.00 & 1963 & [25] \\
\hline $23 \mathrm{~K}$ & French sausage & 1.88 & 41.30 & 1779 & [18] \\
\hline J64 & Dry sausage & 2.05 & 41.06 & 2102 & [23] \\
\hline J156 & Dry sausage & 1.88 & 41.07 & 1817 & [23] \\
\hline $\mathrm{J} 112$ & Dry sausage & 1.98 & 41.04 & 1913 & [23] \\
\hline $\mathrm{J} 54$ & Dry sausage & 2.01 & 41.31 & 1982 & [23] \\
\hline J18 & Dry sausage & 1.82 & 41.00 & 1826 & [23] \\
\hline MFPB16A1401 & Beef carpaccio & 2.04 & 41.09 & 1960 & [23] \\
\hline MFPB19 & Beef carpaccio & 2.06 & 41.05 & 1977 & [23] \\
\hline $\mathrm{J} 160 \times 1$ & Horse meat & 1.90 & 41.07 & 1857 & [23] \\
\hline FAM18311 & $\begin{array}{c}\text { Fermented meat } \\
\text { products }\end{array}$ & 1.95 & 41.3 & 1896 & [26] \\
\hline JXJ41 & Kimchi water & 2.04 & 41.80 & 2010 & This work \\
\hline RS704 & Kimchi water & 1.96 & 41.86 & 1945 & This work \\
\hline DS4 & Kimchi & 2.10 & 41.41 & 1970 & [24] \\
\hline Probio65 & Kimchi & 2.08 & 41.18 & 1984 & [24] \\
\hline WiKim0063 & Kimchi & 2.08 & 41.17 & 1955 & [24] \\
\hline WiKim0072 & Kimchi & 2.03 & 41.17 & 1936 & [24] \\
\hline WiKim0073 & Kimchi & 2.04 & 41.16 & 1913 & [24] \\
\hline WiKim0074 & Kimchi & 2.04 & 41.03 & 1916 & [24] \\
\hline WiKim22 & Kimchi & 2.19 & 40.61 & 1869 & [27] \\
\hline CBA3614 & kimchi & 2.02 & 41.13 & 1910 & [28] \\
\hline MBEL1397 & Kimchi & 1.99 & 41.04 & 1847 & [29] \\
\hline FBL1 & Mukeunji & 2.03 & 41.20 & 1936 & [30] \\
\hline QJSSZ4L10 & $\begin{array}{l}\text { Pickled green } \\
\text { vegetable heart }\end{array}$ & 2.05 & 41.70 & 2075 & This work \\
\hline QJLYJ4L4 & Pickled radish & 1.97 & 41.76 & 1928 & This work \\
\hline СЕСТ9267 & $\begin{array}{c}\text { Fermented } \\
\text { potatos }\end{array}$ & 2.03 & 40.90 & 1972 & [24] \\
\hline СЕСТ9624 & $\begin{array}{c}\text { Fermented } \\
\text { potatos }\end{array}$ & 2.06 & 40.80 & 1986 & [31] \\
\hline ZFM229 & $\begin{array}{l}\text { Fermented } \\
\text { vegetables }\end{array}$ & 2.02 & 41.22 & 1907 & [28] \\
\hline LZ217 & $\begin{array}{l}\text { Fermented } \\
\text { vegetables }\end{array}$ & 2.02 & 41.22 & 1908 & [28] \\
\hline RI-516 & Cacao bean & 1.91 & 41.00 & 1847 & [24] \\
\hline RI-517 & Cacao bean & 1.95 & 41.00 & 1878 & [24] \\
\hline
\end{tabular}




\begin{tabular}{|c|c|c|c|c|c|}
\hline LS25 & $\begin{array}{c}\text { Commercial } \\
\text { product Bitec } \\
\text { LS-25 }\end{array}$ & 2.02 & 40.90 & 1972 & [32] \\
\hline DSM20017 & Sake & 1.91 & 41.1 & 1820 & [24] \\
\hline TMW1.411 & Starter culture & 1.94 & 41.06 & 1859 & [31] \\
\hline LK-145 & $\begin{array}{c}\text { Japanese sake } \\
\text { cellar }\end{array}$ & 1.99 & 41.15 & 1901 & [33] \\
\hline NBRC3541 & Raw moto-shu & 1.94 & 41.10 & 1855 & [34] \\
\hline NBRC15893 & Kimoto & 1.90 & 41.10 & 1822 & [35] \\
\hline ATCC15521 & $\begin{array}{l}\text { Moto, starter of } \\
\text { sake }\end{array}$ & 1.94 & 41.10 & 1835 & Unknown \\
\hline ZFM220 & Raw cow milk & 2.02 & 41.22 & 1909 & [28] \\
\hline ZFM225 & Raw cow milk & 2.02 & 41.22 & 1909 & [28] \\
\hline AMBR8 & $\begin{array}{c}\text { Nasopharyngeal } \\
\text { samples }\end{array}$ & 2.00 & 41.00 & 1923 & [36] \\
\hline RI-493 & Unknown & 1.97 & 40.90 & 1890 & [24] \\
\hline CBA3635 & Unknown & 2.06 & 41.10 & 2002 & [28] \\
\hline L15 & Unknown & 1.98 & 41.00 & 1915 & [28] \\
\hline ye2 & Unknown & 1.99 & 41.10 & 1907 & [28] \\
\hline RI-403 & Unknown & 2.00 & 41.00 & 2032 & [25] \\
\hline RI-404 & Unknown & 1.95 & 40.90 & 1977 & [25] \\
\hline RI-409 & Unknown & 1.99 & 41.00 & 2022 & [25] \\
\hline RI-410 & Unknown & 1.93 & 41.10 & 1949 & [25] \\
\hline RI-412 & Unknown & 1.92 & 41.10 & 1934 & [25] \\
\hline $\begin{array}{c}\text { MGYG-HGUT- } \\
02389\end{array}$ & Unknown & 1.96 & 41.20 & 1813 & Unknown \\
\hline ERR260134-bin.14 & Unknown & 1.52 & 41.20 & - & Unknown \\
\hline NBRC107868 & Unknown & 1.97 & 41.00 & 1920 & Unknown \\
\hline
\end{tabular}

\subsection{Genome Features Prediction and Annotation}

Glimmer 3.02 [37] (http://ccb.jhu.edu/software/glimmer) prediction software was used to predict the $\mathrm{G}+\mathrm{C}$ content of each genome. The Swiss-Prot [38] and RefSeq nonredundant proteins (NR) databases [39] using Diamond software were used to annotate amino acid sequences, with an E-value of 1e-5. Glimmer (http://ccb.jhu.edu/software/glimmer/index.shtml) can predict the coding sequence (CDS) of the genome.

\subsection{Pan-Genome and Core-Genome Analysis}

Using PGAP-1.2.1 software, genomes were examined based on protein sequences, annotation information and nucleotide sequences, and then analyzed according to the Heap's law pan-genome model [40], to calculate the pan-genome of L. sakei. The protein sequence alignment of 68 strains was completed using Orthomcl software, and a Venn diagram was constructed [41]. According to the Clusters of Orthologous Groups (COG) (http://www.ncbi.nlm.nih.gov/COG/) assignments, the functions of the genome encoded proteins were categorized.

\subsection{Average Nucleotide Identity (ANI) Values and Phylogenetic analyses}


The method of calculating ANI was used to average the consistency of homologous genes of each pair for sequences [42]. The python script was used to calculate the ANI value between any two genomes (https://github.com/widdowquinn/pyani) [43] and the obtained matrix is clustered and visualized by $\mathrm{R}$ package heat map software.

Orthomcl-v2.0.9 software was used to extract all orthologous protein sequences of 68 strains and the homologous genes were clustered [44]. MEGA 7.0 software was used to construct the phylogenetic tree of the MAFFT-aligned sequence [45], and modified by Evolgenius (https://evolgenius.info//evolview) [46].

\subsection{Genotype/Phenotype Association Applied to Carbohydrate Metabolism}

The annotation of carbohydrate utilization genes was performed using the Carbohydrate Active Enzyme Database (CAZy) [47]. Thereafter, the annotated results were analyzed by cluster analysis using HemI software [48].

The utilization ability of 14 carbohydrates from 14 L. sakei strains was determined. The carbohydrate stock solution was filtered through a $0.22 \mu \mathrm{m}$ filter membrane, and then added to MRS medium without carbohydrate. Thereafter, $0.5 \%(\mathrm{w} / \mathrm{v})$ bromocresol purple solution was added as an indicator. L. sakei was cultured at $37^{\circ} \mathrm{C}$ for $24 \mathrm{~h}$ with $1 \%$ inoculum in the culture medium, and the change in color was used to judge its utilization. The experiment was repeated three times.

\subsection{Genotype/Phenotype Association Applied to Antibiotic Resistance}

The predicted antibiotic resistance gene information in the genome was obtained by comparing the amino acid sequences of the strains with the comprehensive database of antibiotics antibiotic research database (CARD, http://arpcard.mcmaster.ca) [49]. The strains were clustered using HemI software [48].

The Minimum Inhibitory Concentration (MIC) of 14 antibiotics (chloramphenicol, rifampicin, streptomycin, kanamycin, gentamycin, tetracycline, clindamycin, neomycin, erythromycin, ciprofloxacin and vancomycin) against L. sakei was determined. The double dilution method was used to dilute the antibiotics according to the standard. Diluted antibiotic solutions with different concentrations were added to a sterile 96-well plate, the first well as the blank control, and add $100 \mu \mathrm{L}$ sterile water and MRS culture medium, respectively. $100 \mu \mathrm{L}$ of antibiotic diluent was added to the second to eleventh wells according to the concentration from low to high. The 12th hole was the positive control for strain growth. After activation, L. sakei strain was diluted to $10^{5}-10^{6} \mathrm{CFU} / \mathrm{mL}$, and $100 \mu \mathrm{L}$ of bacterial suspension was added to the 96 -well plate. After culturing at $37^{\circ} \mathrm{C}$ for $24 \mathrm{~h}$, $\mathrm{OD}_{625}$ was determined using an enzyme-labeled instrument to determine the MIC of strain to antibiotics.

\subsection{CRISPR Identification and Characterization of Isolated Strains}

CRISPR loci, CRISPR repeats and spacers in L. sakei were excavated and predicted using CRISPRFinder (https://crisprcas.i2bc.paris-saclay.fr/CrisprCasFinder/Index) [50]. The repeated secondary structure was predicted using RNAfold (http://rna.tbi.univie.ac.at/cgi-bin/RNAWebSuite/RNAfold.cgi) [51]. Phylogenetic analysis was performed based on amino acid sequence and CRISPR repeats of Cas1, Cas2 and Cas9 proteins. MEGA software (version 7.0; Sudhirkumar, Philadelphia, PA., USA) was used to construct the phylogenetic tree.

\section{Results}

\subsection{General Genome Characteristics of Latilactobacillus sakei}

In our previous research, 14 L. sakei strains were isolated from different niches, such as human feces, fermented vegetable products, fermented meat products and kimchi water (Table 1). Combined with the genome information of 54 L. sakei strains published in 
NCBI GenBank database, the genomes of $68 \mathrm{~L}$. sakei strains were compared and analyzed (Table 1). The genome size of 68 L. sakei strains ranged from $1.54 \mathrm{Mb}$ (L. sakei ERR260134bin.14) to $2.19 \mathrm{Mb}$ (L. sakei WiKim22) with an average size of $1.98 \mathrm{Mb}$. The average G+C content was $41.22 \%$, ranging from $40.61 \%$ for L. sakei WiKim22 to $42.03 \%$ for L. sakei QAHLA3L8. The average predicted coding sequences (CDSs) of each genome was 1923, ranging from 1779 for L. sakei $23 \mathrm{~K}$ to 2142 for L. sakei FXJWS8M1.

\subsection{Pan-Genome and Core Genes of Latilactobacillus sakei}

To study the genetic diversity of L. sakei, the pan genome and core genes were analyzed. The functional relationship between the number of core genes and pan genes and the number of sequencing strains was plotted (Figure 1a). The results showed that with an increase in the number of L. sakei strains, the number of pan-genes increased continuously, and the number of core genes tended to be stable. When the 68th strain was added, the number of ubiquitin genes was stable at 5983, and the number of core genes reached 993. The pan-genome curve shows an asymptotic trend, which may indicate that L. sakei has an open pan-genome. The specific core genes and homologous core genes of L. sakei strains were analyzed, and Wayne diagram was drawn (Figure 1b). 68 L. sakei strains had 1099 common core genes, and each strain had 4-160 unique core genes. Functional analysis of the core genes of L. sakei, revealed that the core genome includes replication, transcription, translation, nucleotide metabolism, carbohydrate metabolism, amino acid metabolism, lipid metabolism and other related genes. Among them, genes related to carbohydrate metabolism accounted for approximately $7.53 \%$ of core functional genes, $5.68 \%$ of core functional genes were related to amino acid metabolism, however, $28.24 \%$ of core genome functions are unknown (Figure 1c).
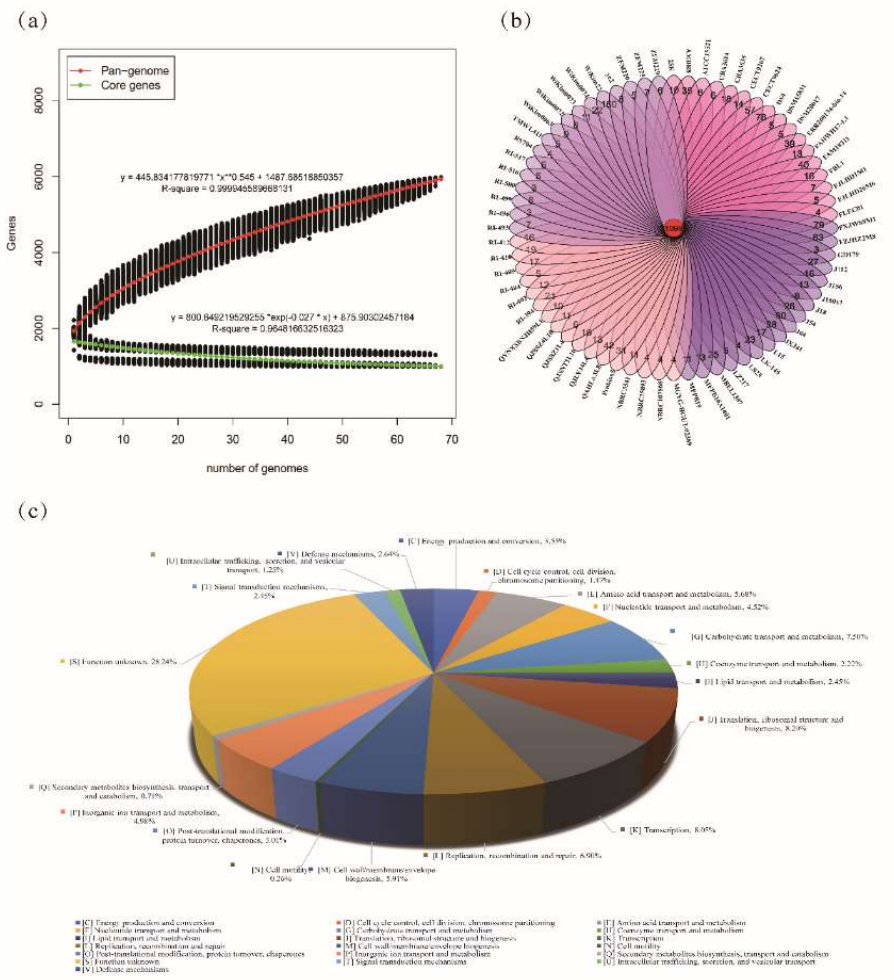

Figure 1. Pan- and core genes of Latilactobacillus sakei. (a) Pan-genome and core genome; (b) Venn diagram displaying the unique and core genes; (c) Functional assignment of the core genome based on the COG database.

\subsection{ANI and Phylogenetic Analyses of Latilactobacillus sakei}

Average nucleotide identity (ANI) is a classic method that can analyze unique species or potential subspecies within the same strain. The generally accepted ANI boundary 
value is $95-96 \%$ [52]. When the ANI value is less than this boundary value, the strain may be a potential subspecies. Among the 68 L. sakei strains, except L. sakei DS4, the ANI values of the other strains were higher than 97\% (Figure 2a). The ANI value between strain DS4 and other L. sakei is only $92 \%-93 \%$, indicating that L. sakei DS4 may be a potential subspecies. However, this conjecture has not been confirmed by research, and whether L. sakei DS4 is a potential subspecies needs further discussion.

(a)

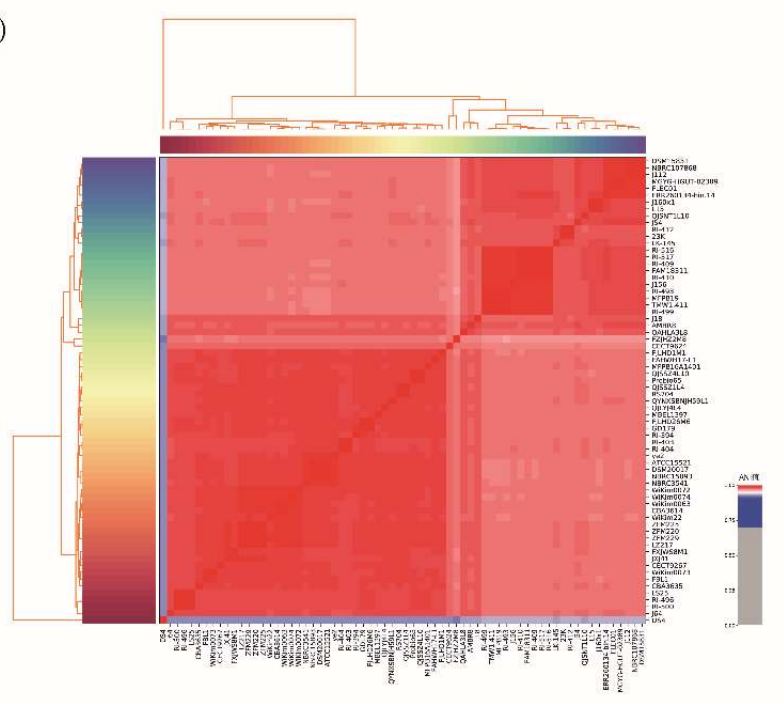

(b)

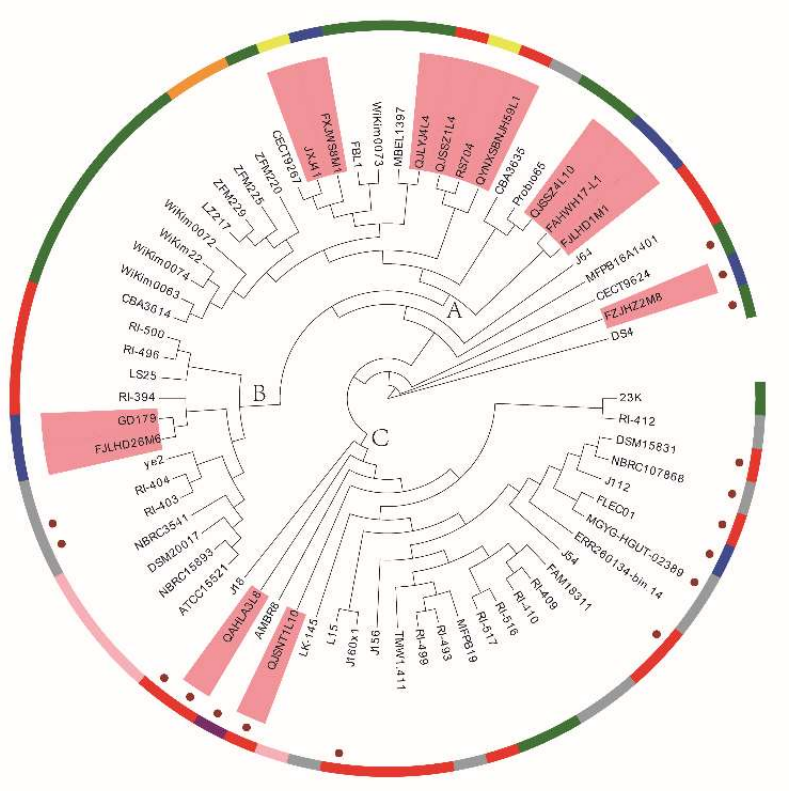

Figure 2. The Average Nucleotide Identity (ANI) and phylogenetic analysis of Latilactobacillus sakei. (a) Heatmap showing the ANI value among 68 L. sakei strains; (b) Phylogenetic tree based on orthologous genes. The salmon area is the strain isolated in our laboratory. Brown circles represent strains with complete CRISPR-Cas system. The peripheral blue band represents strains from feces, the red band represents strains from fermented meat products, the green band represents strains from fermented vegetables, the pink band represents strains from fermented sake, the yellow band represents strains from kimchi water, the orange band represents strains from milk, and the source of gray band separation is unknown.

The phylogenetic tree was constructed based on homologous genes of 68 L. sakei strains to explore the phylogenetic relationships (Figure $2 b$ ). The phylogenetic tree was divided into two large clades, among which L. sakei DS4 was divided into one, which may 
be related to its low ANI value. The remaining strains were mainly divided into three evolutionary clades (A-C), and the strains isolated in our laboratory exist in all three evolutionary clades. In clade A, three strains were from feces, and the other six strains were from fermented products. Clade B was isolated from feces, whereas clade $C$ was isolated from fermented meat products.

\subsection{Genotype/Phenotype Association Analysis for Carbohydrates Utilization in Latilactobacillus sakei}

To understand the carbohydrate utilization ability of L. sakei, the sequencing genomes of $14 \mathrm{~L}$. sakei strains isolated in our laboratory were analyzed using the CAZy database. There are four carbohydrate active enzyme families in 14 L. sakei strains, including auxiliary activity (AAs) families, carbohydrate esterase (CEs) families, glycoside hydrolase (GHs) families and glycosyltransferases (GTs) families. The GH family is the most abundant enzyme in L. sakei, followed by the GT family, and the number of carbohydrate active enzyme genes encoding the CE and AA families decreased in turn (Figure 3a). Among these strains, AA10 (lytic cellulose monooxygenase (EC 1.14.99.56)), CE1 (feruloyl esterase (EC 3.1.1.73)), GH1 ( $\beta$-glucosidase (EC 3.2.1.21)), GH2 ( $\beta$-galactosidase (EC 3.2.1.23)), GH13_29 ( $\alpha$-amylase (EC 3.2.1.1)), GT4 (sucrose synthase (EC 2.4.1.13)) and other 16 active genes were found in all strains, whereas the distribution of the remaining genes was different in each strain (Figure 3b). For example, CE4 (acetyl xylan esterase (EC 3.1.1.72)) only exists in L. sakei FJLHD1M1, and AA6 (1,4-benzoquinone reductase (EC. 1.6.5.6)) was absent in L. sakei FXJWS8M1. GH1, GH2 and GH109 account for a high proportion in GH family, which are related to the catabolism of carbohydrates such as lactose and mannose. In addition, GT2 and GT4 accounted for a higher proportion in the GT family. Based on the clustering results of carbohydrate utilization active enzymes of L. sakei, it was found that there was no evident regularity between the clustering results of various strains and the host and geographical sources, indicating that there is diversity in the carbohydrate utilization ability of L. sakei.
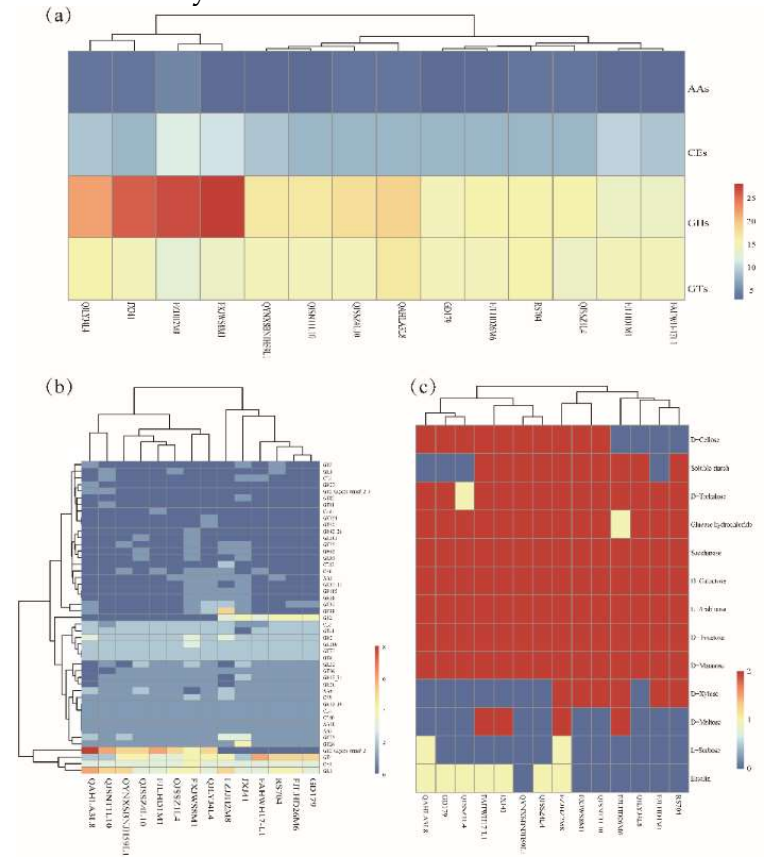

Figure 3. Genotype-phenotype analysis of carbohydrate utilization of Latilactobacillus sakei. (a) Comparative heat map of the number of genes in four families of carbohydrate active enzymes of different strains of L. sakei; (b)Thermographic analysis of carbohydrate enzyme activity related genes of L. sakei; (c) Utilization ability of 13 carbohydrates by L. sakei.

To verify the genotype, the ability of L. sakei to utilize 13 carbohydrates was determined. All strains utilized glucose hydrochloride, D-mannose, D-fructose, L-arabinose, D-galactose and sucrose, but have strain specificity for the utilization of D-maltose, D- 
xylose, soluble starch, L-sorbose, D-trehalose, D-cellobiose and escin (Figure 3c). The metabolism of D-galactose is responsible fot $\beta$-galactosidase, which belongs to GH2 and GH42 families. It is not difficult to find that all L. sakei containing GH2 or GH42 gene can utilize D-galactose. In addition, $\beta$-mannosidase in $\mathrm{GH} 2$ is responsible for mannose catabolism, promoting the utilization of mannose by $14 \mathrm{~L}$. sakei strains. All strains contained GH13_29 family enzymes, which have $\alpha$-amylase related to starch hydrolysis and trehalose -6- phosphate hydrolase related to trehalose hydrolysis. However, four strains did not show the ability to utilize soluble starch, and one strain showed the ability to utilize trehalose, which may be due to the lack of expression of GH13_29 in the four strains.

\subsection{Genotype/Phenotype Association Analysis for Antibiotic Resistance in Latilactobacillus sakei}

The genome of L. sakei was annotated using CRAD database. The annotation results showed that 117 resistance genes were predicted in 14 L. sakei strains (Figure 4a), including macrolide resistance genes $(m a c B)$, glycopeptide resistance genes (vanRF, vanRI), aminoglycoside resistance genes (baeR, baeS), fluoroquinolone resistance genes $(m f d, m f p A)$, lincoamide resistance genes $(\operatorname{lm} r B, \operatorname{lm} r D)$, streptogramin resistance genes (vat $B$, vat $F)$, tetracycline resistance genes (emrY, rpsJ, tet $M$, tet $B(P)$, tet $T)$ and rifamycin resistance genes $(r p h B)$. By comparing the total number of resistance genes of 14 L. sakei isolates from different sources, it was found that the resistance genes of L. sakei isolated from feces and kimchi water were higher than those isolated from fermented products (Figure 4b). Among them, the number of resistance genes of L. sakei FZJHZ2M8 was the highest (127), whereas that of L. sakei QJLYJ4L4 and L. sakei QYNXSBNJH59L1 was the least (104). In addition, the distribution of some resistance genes appears to be related to the isolated sources. For example, rifamycin resistance gene $r p h B$ and tetracycline resistance gene tet $B(60)$ exist in all strains isolated from fermented products and a few strains isolated from feces. However, the resistance genes $d$ fr $A 8$ and $\beta$-lactam $C M Y-73$ were mostly found in the strains isolated from feces and kimchi water; however, none of the strains isolated from fermented products contained these genes (Figure 4a). 


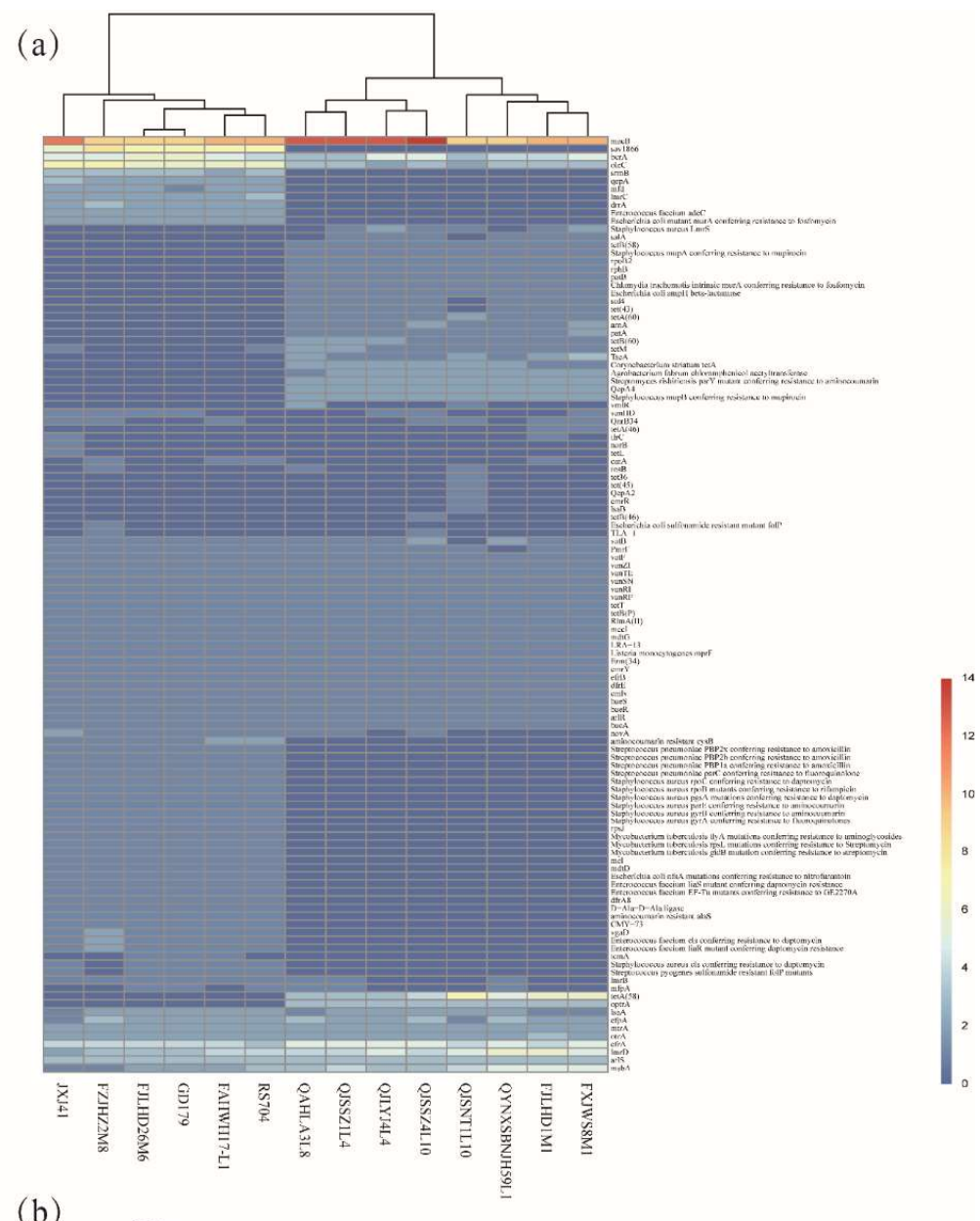

(b)

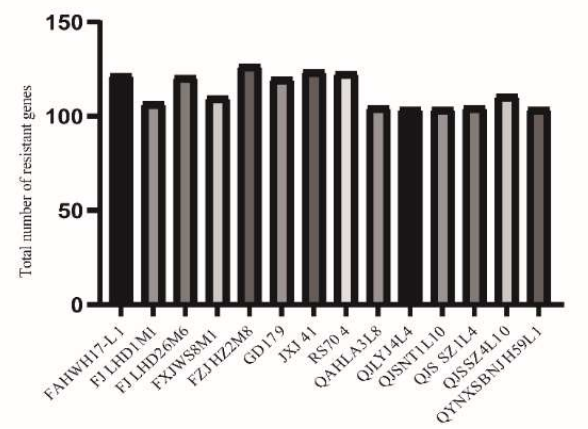

Figure 4. Genotype-phenotype analysis of antibiotic resistance of Latilactobacillus sakei. (a) Clustering heat map analysis of antibiotic resistance genes in L. sakei; (b) Total number of resistance genes of 14 L. sakei strains.

Furthermore, the genetic traits of $14 \mathrm{~L}$. sakei strains were matched and the tolerance of $L$. sakei to 10 antibiotics such as erythromycin, vancomycin, streptomycin, kanamycin, gentamycin, neomycin, tetracycline, rifampicin, ciprofloxacin and clindamycin were analyzed (Table 2). There are glycopeptide resistance genes and aminoglycoside resistance genes in all strains; therefore, all strains have good tolerance to typical glycopeptide antibiotics such as vancomycin and aminoglycoside antibiotics such as streptomycin, kanamycin and gentamycin. Macrolide resistance genes are abundant in 14 strains of L. sakei and the strains have good tolerance to erythromycin, except for L. sakei QJSNT1L10. TetT and $\operatorname{tet} B(P)$ genes related to tetracycline resistance exist in all strains; however, six strains are sensitive to tetracycline, which may indicate that tet $T$ and $\operatorname{tet} B(P)$ are not the key genes of tetracycline resistance. 
Table 2 MIC of different antibiotics for Latilactobacillus sakei.

\begin{tabular}{|c|c|c|c|c|c|c|c|c|c|c|}
\hline \multirow[b]{2}{*}{ Strain } & \multicolumn{10}{|c|}{ Concentration $(\mu \mathrm{g} / \mathrm{mL})$} \\
\hline & $\mathrm{RD}$ & S & K & $\mathrm{CN}$ & $\mathrm{TE}$ & DA & $\mathrm{N}$ & $\mathrm{E}$ & $\mathrm{CIP}$ & VA \\
\hline FJLHD1M1 & 1 & 256 & 256 & 1024 & 2 & 0.5 & 256 & 1 & 128 & 128 \\
\hline GD17-9 & 1 & 256 & 128 & 1024 & 2 & 0.5 & 256 & 1 & 64 & 128 \\
\hline FAHWH17-L1 & 0.5 & 512 & 256 & 1024 & 16 & 2 & 256 & 4 & 128 & 128 \\
\hline JXJ4-1 & 1 & 512 & 128 & 1024 & 64 & 0.5 & 64 & 4 & 128 & 128 \\
\hline QYNXSBNJH59L1 & 0.25 & 512 & 256 & 1024 & 4 & 0.5 & 256 & 2 & 128 & 128 \\
\hline QJSSZ4L10 & 0.5 & 512 & 512 & 1024 & 8 & 0.25 & 128 & 4 & 128 & 128 \\
\hline QJLYJ4L4 & 0.5 & 256 & 128 & 256 & 0 & 0.25 & 256 & 8 & 128 & 128 \\
\hline QJSSZ1L4 & 1 & 512 & 256 & 1024 & 32 & 0.5 & 256 & 1 & 128 & 128 \\
\hline QAHLA3L8 & 1 & 256 & 256 & 1024 & 32 & 0.5 & 256 & 2 & 64 & 128 \\
\hline FJLHD26M6 & 0.25 & 256 & 256 & 512 & 4 & 0.125 & 32 & 2 & 128 & 128 \\
\hline FXJWS8M1 & 8 & 256 & 256 & 1024 & 2 & 0.25 & 128 & 1 & 64 & 128 \\
\hline FZJHZ2M8 & 1 & 256 & 512 & 256 & 64 & 4 & 256 & 2 & 128 & 128 \\
\hline RS704 & 2 & 256 & 128 & 512 & 64 & 0.5 & 256 & 2 & 64 & 128 \\
\hline QJSNT1L10 & 0.125 & 128 & 128 & 1024 & 64 & 0.0625 & 128 & 0.5 & 32 & 128 \\
\hline
\end{tabular}

RD: Rifampicin; S: Streptomycin; K: Kanamycin; CN: Gentamicin; TE: Tetracycline; DA: Clindamycin; N: Neomycin; E: Erythrocin; CIP: Ciprofloxacin; VA: Vancomycin.

\subsection{Prediction of CRISPR-Cas Systems in Latilactobacillus sakei}

Fourteen $14 \mathrm{~L}$. sakei strains isolated from our laboratory were analyzed using the CRISPR-Cas system, and 13 strains were identified as CRISPRs. However, only genomes with evidence levels above 1 were considered in this study due to differences in evidence level. Moreover, CRISPR, an orphan without Cas protein, was ignored because it could not silence foreign DNA. Two strains with complete CRISPR-Cas system were isolated from fermented products (QJSNT1L10 and QAHLA3L8), and one strain was isolated from baby feces (FZJHZ2M8), all of which belonged to class IIA or IIC, including Cas1, Cas2, Cas9 and Csn2 (Table 3). To further explore the distribution characteristics of the CRISPRCas system of $L$. sakei, the CRISPR-Cas system of 54 strains of $L$. sakei on NCBI was predicted. Among the 54 strains, 13 strains had complete CRISPR-Cas system, and these systems were all IIA or IIC. Most of these 14 strains were isolated from fermented products (Table 3).

Table 3. CRISPR-Cas systems in Latilactobacillus sakei

\begin{tabular}{|c|c|c|c|c|c|c|c|}
\hline strains & Repeat sequence $\quad\left(5^{\prime}-3^{\prime}\right)$ & $\begin{array}{l}\text { CAS_- } \\
\text { Subtype }\end{array}$ & $\begin{array}{l}\text { Repeat } \\
\text { Length }\end{array}$ & $\begin{array}{l}\text { No. } \\
\text { Spacer }\end{array}$ & Cas1 & Cas2 & Cas9 \\
\hline \multirow[t]{2}{*}{ FZJHZ2M8 } & GTTTTAGAAGAGTATCAAATCA & IIA & 36 & 16 & $\mathrm{Y}$ & $\mathrm{Y}$ & $\mathrm{Y}$ \\
\hline & $\begin{array}{l}\text { ATTTCATCTTAACCGAATGTTGA } \\
\text { AGGAAACAATAGC }\end{array}$ & IIC & 36 & 71 & $\mathrm{Y}$ & $\mathrm{N}$ & Y \\
\hline
\end{tabular}




\begin{tabular}{|c|c|c|c|c|c|c|c|}
\hline QJSNT1L10 & $\begin{array}{l}\text { GTTTTAGAAGAGTATCAAATCA } \\
\text { ATGAGTAGTTCAAC }\end{array}$ & IIA & 36 & 18 & $\mathrm{~N}$ & $\mathrm{~N}$ & $\mathrm{~N}$ \\
\hline QAHLA3L8 & $\begin{array}{l}\text { GTTGAACTACTCATTGATTTGAT } \\
\text { АСТСТTCTAAAAC }\end{array}$ & IIA & 36 & 33 & Y & $\mathrm{N}$ & Y \\
\hline DS4 & $\begin{array}{l}\text { GCTATTGTTTCCTTCAACATTCG } \\
\text { GTTAAGATGAAAT }\end{array}$ & IIC & 36 & 26 & $\mathrm{Y}$ & $\mathrm{Y}$ & $\mathrm{Y}$ \\
\hline $\mathrm{J} 18$ & $\begin{array}{l}\text { GCTATTGTTTCCTTCAACATTCG } \\
\text { GTTAAGATGAAAC }\end{array}$ & IIC & 36 & 92 & Y & $\mathrm{Y}$ & Y \\
\hline СЕСТ9624 & $\begin{array}{l}\text { GTTTTAGAAGAGTATCAAATCA } \\
\text { ATGAGTAGTTCAAC }\end{array}$ & IIA & 36 & 8 & Y & $\mathrm{Y}$ & $\mathrm{Y}$ \\
\hline & $\begin{array}{l}\text { ATTTCATCTTAACCGAATGTTGA } \\
\text { AGGAAACA }\end{array}$ & IIC & 31 & 2 & $\mathrm{Y}$ & $\mathrm{Y}$ & Y \\
\hline $\mathrm{J} 54$ & $\begin{array}{l}\text { GTTGAACTACTCATTGATTTGAT } \\
\text { АСТCTTCTAAAAC }\end{array}$ & IIA & 36 & 29 & $\mathrm{Y}$ & $\mathrm{Y}$ & $\mathrm{Y}$ \\
\hline $\mathrm{J} 112$ & $\begin{array}{l}\text { GTTTTAGAAGAGTATCAAATCA } \\
\text { ATGAGTAGTTCAAC }\end{array}$ & IIA & 36 & 14 & Y & $\mathrm{Y}$ & $\mathrm{Y}$ \\
\hline FLEC01 & $\begin{array}{l}\text { GTTGAACTACTCATTGATTTGAT } \\
\text { ACTCTTCTAAAAC }\end{array}$ & IIA & 36 & 35 & $\mathrm{Y}$ & $\mathrm{Y}$ & $\mathrm{Y}$ \\
\hline $\mathrm{J} 160 \times 1$ & $\begin{array}{l}\text { GTTGAACTACTCATTGATTTGAT } \\
\text { АСТCTTCTAAAAC }\end{array}$ & IIA & 36 & 24 & $\mathrm{Y}$ & $\mathrm{Y}$ & $\mathrm{Y}$ \\
\hline MGYG-HGUT-02389 & $\begin{array}{l}\text { GTTGAACTACTCATTGATTTGAT } \\
\text { ACTCTTCTAAAAC }\end{array}$ & IIA & 36 & 35 & $\mathrm{Y}$ & $\mathrm{Y}$ & $\mathrm{Y}$ \\
\hline DSM15831 & $\begin{array}{l}\text { AGTTGAACCACTCATTGATTTGA } \\
\text { TACTCTTCTAAAAC }\end{array}$ & IIA & 37 & 11 & $\mathrm{~N}$ & $\mathrm{~N}$ & $\mathrm{~N}$ \\
\hline RI-403 & $\begin{array}{l}\text { GTTTTAGAAGAGTATCAAATCA } \\
\text { ATGAGTGGTTCAAC }\end{array}$ & IIA & 36 & 40 & $\mathrm{~N}$ & $\mathrm{~N}$ & $\mathrm{~N}$ \\
\hline RI-404 & $\begin{array}{l}\text { GTTTTAGAAGAGTATCAAATCA } \\
\text { ATGAGTGGTTCAAC }\end{array}$ & IIA & 36 & 37 & $\mathrm{Y}$ & $\mathrm{Y}$ & $\mathrm{Y}$ \\
\hline AMBR8 & $\begin{array}{l}\text { GTTGAACCACTCATTGATTTGAT } \\
\text { АСТCTTCTAAAAC }\end{array}$ & IIA & 36 & 23 & $\mathrm{Y}$ & $\mathrm{Y}$ & $\mathrm{Y}$ \\
\hline NBRC107868 & $\begin{array}{l}\text { GTTTTAGAAGAGTATCAAATCA } \\
\text { ATGAGTGGTTCAACT }\end{array}$ & IIA & 37 & 11 & $\mathrm{~N}$ & $\mathrm{~N}$ & $\mathrm{~N}$ \\
\hline
\end{tabular}

$\mathrm{Y}$ : present; $\mathrm{N}$ : none. 
By analyzing the number of spacer sequences of CRISPR loci with different subtypes of L. sakei (Figure 5a), the number of interval sequences of type IIC loci is quite different, up to 92 and at least 2. Repeat sequences were explored by their secondary structure (Figure $5 b-d)$. According to the repeated sequences, it is predicted that there are two typical secondary structures in subtype IIA (Figure $5 b-c$ ) and one typical secondary structure in subtype IIC (Figure 5d). Repeated sequence is a typical stem-loop stable structure, which contains a large loop and a small loop at both ends. The phylogenetic tree was constructed with Cas1, Cas2 and Cas9 protein genes and we can find that Cas1, Cas2 and Cas9 protein genes of subtype IIA and IIC have strict single-line inheritance, but there were a few exceptions (Figure 6a-c).

(a)

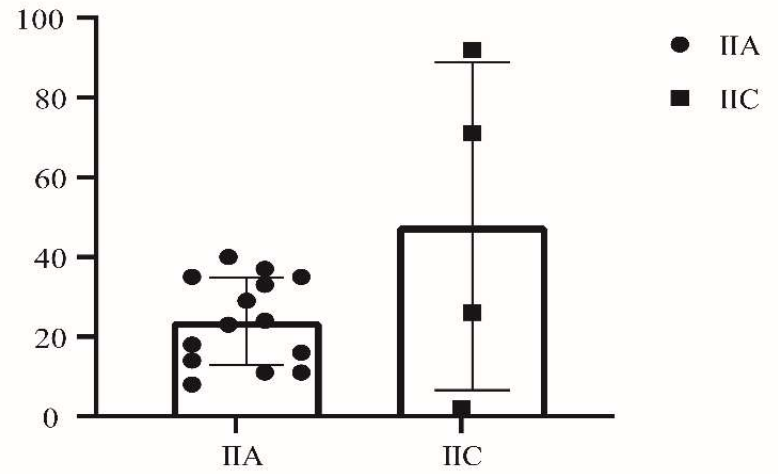

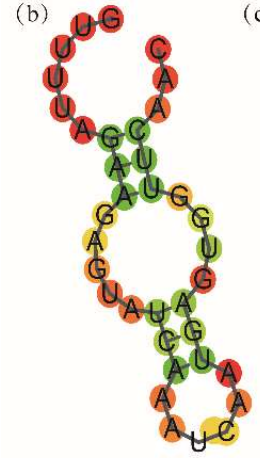

IIA

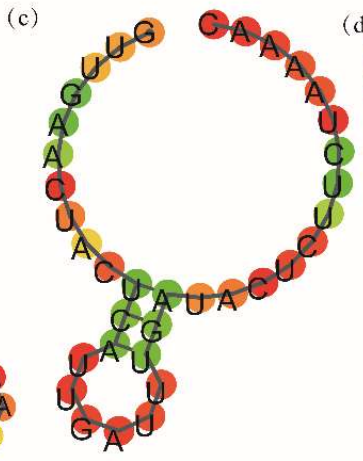

IIA

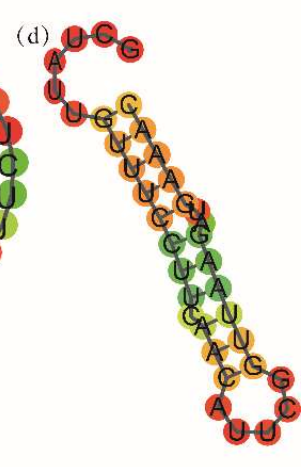

IIC

Figure 5. Prediction of CRISPR-Cas Systems in Latilactobacillus sakei. (a) Number of spacer sequences of CRISPR loci in different subtypes of Latilactobacillus sakei; (b-d) Predicted RNA secondary structures of CRISPR DR in Latilactobacillus sakei.
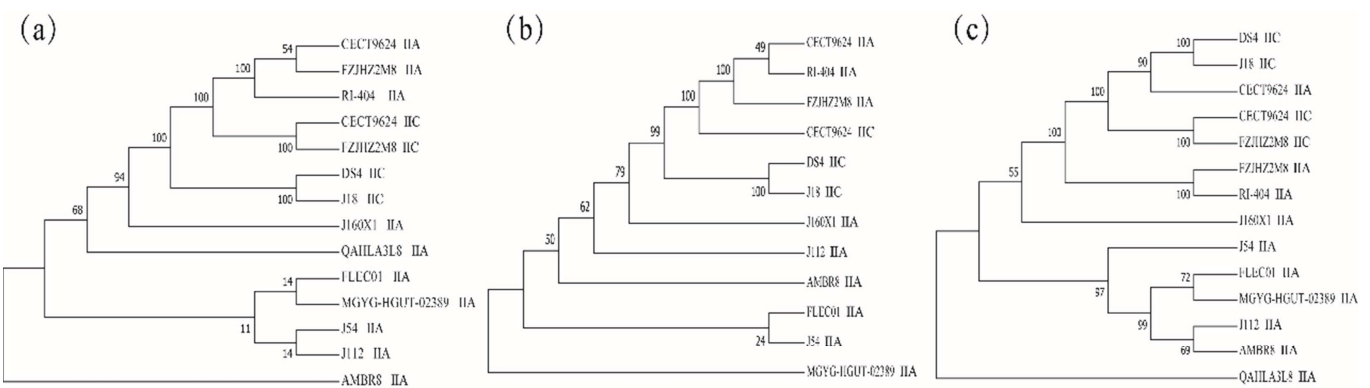

Figure 6. CRISPR-Cas phylogenetic analyses for Latilactobacillus. sakei. (a) Phylogenetic tree based on the Cas1 protein; (b) Phylogenetic tree based on the Cas2 protein; (c) Phylogenetic tree based on the Cas9 protein.

\section{Discussion}

Latilactobacillus sakei is a potential candidate probiotic that exists in many niches. A study shows that L. sakei can not only be used as a starter [53] and biological protective 
agent [54] in the food industry, and has a variety of probiotic functions, such as improving metabolic syndrome such as obesity [55], improving immunity and relieving atopic dermatitis [56], and alleviating inflammatory reactions in colitis mice [57]. The development of genomic tools, it provides strong support for diversity analysis of strains. However, there are few studies on the genome diversity of L. sakei, and the isolation sources of strains are relatively single. In this study, the genetic diversity and functional diversity of 68 L. sakei strains from different niches were analyzed using 14 L. sakei strains isolated in our laboratory and 54 strains with published genetic information on NCBI.

The average genome size of 68 strains of $L$. sakei was $1.98 \mathrm{Mb}$, and the average GC content was $41.22 \%$, which is consistent with the study of Eisenbach et al. [58]. The average GC content is lower than that of Ligilactobacillus ruminis [59], Lacticaseibacillus casei [60] and other lactobacillus which belonging to free living and nomadic lifestyle, although higher than that of Pediococcus pentosaceus [61] and Lactobacillus crispatus [62]. The pan-genome and core genome showed that $L$. sakei has an open pan-genome. In addition, the function and translation, defense mechanism and general function prediction of L. sakei were revealed by annotating the core genes.

ANI is a classical index, used to distinguish whether a particular strain belongs to the same species, and commonly takes a 95\% threshold as the species boundary [52]. Except for L. sakei DS4, the ANI value of other strains was more than 97\%, whereas that of DS4 was only $92 \%$, which reveals that DS4 may be a potential subspecies. Based on the phylogenetic tree of homologous gene sequence similarity, 68 L. sakei strains were divided into two major clades and three main sub-clades. There was no evident correlation between each branch and the isolated source.

To further understand the fermentation ability of L. sakei using carbohydrates, the genome of 14 strains of L. sakei isolated in our laboratory was analyzed and compared using CAZymes technology. GH family enzymes, including GH1, GH2, GH42 and GH73, are the most abundant carbohydrate enzymes in L. sakei. These enzymes are involved in the catabolism of various carbon compounds, such as soluble starch, mannose, xylose, glucose, trehalose, maltose and galactose, consistent with the results of Eisenbach et al. [58]. Aside from the corresponding hydrolases, the phosphotransferase system will also affect the utilization of carbohydrates. Chaillou et al. [18] showed that there are phosphotransferase systems of glucose, mannose, fructose, sucrose and trehalose in L. sakei, which provides a stronger basis for utilizing these carbohydrates by L. sakei. GH25, as lysozyme [63], participates in peptidoglycan and cell wall catabolism, which can promote cell division and defense. $\beta$-N-acetylglucosamine is encoded by GH73, and the $\beta-1,4$ glycosidic bond between $\mathrm{N}$-acetylmuramic acid and $\mathrm{N}$-acetylglucosamine of bacterial cell wall peptidoglycan is cleaved by it [64]. These results provide a competitive advantage for the survival of L. sakei. Microbes also produce a special enzyme called carbohydrate esterase (CE) to deacetylate hemicellulose and pectin units of plant polysaccharides [65]. CE is mainly divided into pectin deacetylating CE and hemicellulose deacetylation. L. sakei is rich in the CE1 family, which is an acetyl xylan esterase.

Owing to their intrinsic and nontransmissible characteristics, many LABs have high antibiotic susceptibility [66]. All 14 strains of L sakei isolated in our study have glycopeptide resistance genes; therefore, they have good tolerance to vancomycin, a typical antibiotic of glycopeptide. This is consistent with the results of Georgieva et al. [67], who found that heterofermentative lactic acid bacteria are resistant to vancomycin. In addition, $14 L$. sakei strains have glycoside resistance genes, which are resistant to streptomycin, kanamycin and gentamicin, which are inherent properties of lactobacillus [68]. There were 13 tetracycline resistance genes in the studied L. sakei, however, six strains were sensitive to tetracycline, among which four strains were isolated from fecal samples. The resistance of most bacteria to tetracycline seems to be obtained horizontally, and the tet gene encoding drug resistance is highly mobile because it is located on conjugated transposons [69]. Most tet genes encode tetracycline resistance efflux proteins, a part of the major facilitator (MFS) of transporters. These proteins are bound by membranes and exchange protons with 
tetracycline cation complexes under concentration gradient [70], which reduces the concentration of tetracycline in cells, thus protecting ribosomes in cells.

CRISPR and Cas combine to form the CRISPR-Cas system, providing adaptive immunity to bacterial invasive components [71]. Sixteen strains of 68 L. sakei strains were identified to have a complete CRISPR-Cas system, including 14 subtypes of IIA and 4 subtypes of IIC. This is consistent with the results of Ilkkan et al. [28] that subtype IIA is the main CRISPR-Cas subtype in L. sakei. In Latilactobacillus curvatus, Loigolactobacillus rennini, Pediococcus damnosus and Secundilactobacillus paracollinoides, which exist in a similar niche to L. sakei, highly similar structures of type II-A CRISPR/Cas and similar Cas protein sequences were also found [31]. The activity of the CRISPR system is reflected by the number of spacer sequences, and continuous acquisition of spacer sequences has been proven in the active CRISPR-Cas system [72]. It can be inferred from the number of spacer sequences that the IIC subtype of L. sakei is more active and can better resist the insertion of foreign genes. The CRISPR-Cas structure can be used in genetic engineering, and its great potential has been proven, particularly Cas9 nuclease [73]. Cas protein is used as a programmable nuclease that is used for efficient and accurate genome editing in various fields of medicine, research and biotechnology. The new possibility of Cas9-mediated genome editing is attributed to the identification of new PAMs(g/a) AAA (for type II-A) and (a/g) (c/t) AC (for type II-C CRISPR Cas system), as well as localized tracrRNAs [31]. Therefore, the application potential of L. sakei Cas nuclease requires further study.

\section{Conclusions}

In this study, comparative genomics was used to analyze the genomes of 68 Latilactobacillus sakei strains, which provided a basis for analyzing the functional genes of this species. The results showed that niche affected the antibiotic resistance of L. sakei, and the strains from feces and pickle water had more abundant antibiotic resistance genes, and its genetic diversity is also reflected in carbohydrate utilization and some immune/competition related factors (CRISPR). Genome sequencing and genetic analysis in this study helped understand the biotechnology potential of L. sakei and promote its future development as a protective agent/starter and a therapeutic agent for microbial related diseases.

Author Contributions: Writing-Original Draft, Ying Chen; Writing-Reviewing and Editing, Nan Li, Shenxi Zhao and Chuan Zhang; Data curation, Formal analysis, Methodology, Leilei Yu, Qixiao Zhai, Nanzhen Qiao and Hui Duan; Supervision, Yue Xiao, Bowen Yan, Leilei Yu; Project administration, Fengwei Tian; Funding acquisition, Jianxin Zhao, Qixiao Zhai, Leilei Yu and Wei Chen.

Funding: This work was supported by the National Natural Science Foundation of China (32001665), the Natural Science Foundation of Jiangsu Province (BK20180603), the National Natural Science Foundation of China (U1903205,31820103010,31772090), the Key Scientific and Technological Research Projects in the Key Areas of the Xinjiang Production and Construction Corps (2018AB010), the Postdoctoral Science Foundation of China (2018M642166), the General Financial Grant from the Jiangsu Postdoctoral Science Foundation (2018K016A), the Shanghai Engineering Research Center of Dairy Biotechnology(19DZ2281400), the National first-class discipline program of Food Science and Technology (JUFSTR20180102), Collaborative innovation center of food safety and quality control in Jiangsu Province.

Conflicts of Interest: The authors declare no conflict of interest.

\section{References}

1. Katagiri, H.; Kitahar, K. The characteristics of the lactic acid bacteria isolated from moto, yeast mashes for sake' manufacture. J. Ceram. Soc. Jpn. 1934, 10, 153-154, doi.org/10.1080/03758397.1934.10857096.

2. Moroni, A.V.; Arendt, E.K.; Bello, D.F. Biodiversity of lactic acid bacteria and yeasts in spontaneously-fermented buckwheat and teff sourdoughs. Food Microbiol. 2011, 28, 497-502, doi:10.1016/j.fm.2010.10.016.

3. Chaillou, S.; Daty, M.; Baraige, F.; Dudez, A.M.; Anglade, P.; Jones, R.; Alpert, C.A.; Champomier-Verges, M.C.; Zagorec, $\mathrm{M}$. Intraspecies genomic diversity and natural population structure of the meat-borne lactic acid bacterium Lactobacillus sakei. Appl. Environ. Microbiol. 2009, 75, 970-980, doi:10.1128/AEM.01721-08. 
4. Chaillou, S.; Chaulot-Talmon, A.; Caekebeke, H.; Cardinal, M.; Christieans, S.; Denis, C.; Desmonts, M.-H.; Dousset, X.; Feurer, C.; Hamon, E., et al. Origin and ecological selection of core and food-specific bacterial communities associated with meat and seafood spoilage. ISME. J. 2015, 9, 1105-1118, doi:10.1038/ismej.2014.202.

5. Dal Bello, F.; Walter, J.; Hammes, W.P.; Hertel, C. Increased complexity of the species composition of lactic acid bacteria in human feces revealed by alternative incubation condition. Microb. Ecol. 2003, 45, 455-463, doi:10.1007/s00248-003-2001-z.

6. Carvalho, K.G.; Bambirra, F.H.S.; Nicoli, J.R.; Oliveira, J.S.; Santos, A.M.C.; Bemquerer, M.P.; Miranda, A.; Franco, B. Characterization of multiple antilisterial peptides produced by sakacin P-producing Lactobacillus sakei subsp. sakei 2a. Arch. Microbiol. 2018, 200, 635-644, doi:10.1007/s00203-018-1477-3.

7. Mathiesen, G.; Huehne, K.; Kroeckel, L.; Axelsson, L.; Eijsink, V.G. Characterization of a new bacteriocin operon in sakacin P-producing Lactobacillus sakei, showing strong translational coupling between the bacteriocin and immunity genes. Appl. Environ. Microbiol. 2005, 71, 3565-3574, doi:10.1128/AEM.71.7.3565-3574.2005.

8. Winkelströter, L.K.; Gomes, B.C.; Thomaz, M.R.S.; Souza, V.M.; De Martinis, E.C.P. Lactobacillus sakei 1 and its bacteriocin influence adhesion of Listeria monocytogenes on stainless steel surface. Food Control. 2011, 22, 1404-1407, doi:10.1016/j.foodcont.2011.02.021.

9. Gao, Y.; Jia, S.; Gao, Q.; Tan, Z. A novel bacteriocin with a broad inhibitory spectrum produced by Lactobacillus sake C2, isolated from traditional Chinese fermented cabbage. Food Control. 2010, 21, 76-81, doi:10.1016/j.foodcont.2009.04.003.

10. Gao, Y.; Li, D.; Liu, X. Effects of Lactobacillus sakei C2 and sakacin C2 individually or in combination on the growth of Listeria monocytogenes, chemical and odor changes of vacuum-packed sliced cooked ham. Food Control. 2015, 47, 27-31, doi:10.1016/j.foodcont.2014.06.031.

11. Najjari, A.; Boumaiza, M.; Jaballah, S.; Boudabous, A.; Ouzari, H.I. Application of isolated Lactobacillus sakei and Staphylococcus xylosus strains as a probiotic starter culture during the industrial manufacture of Tunisian dry-fermented sausages. Food Sci. Nutr. 2020, 8, 4172-4184, doi:10.1002/fsn3.1711.

12. Moon, S.H.; Moon, J.S.; Chang, H.C. Rapid manufacture and quality evaluation of long-term fermented kimchi (mukeunji) using Lactobacillus sakei SC1. Food Sci. Biotechnol. 2015, 24, 1797-1804, doi:10.1007/s10068-015-0234-5.

13. Hong, Y.F.; Kim, H.; Kim, H.R.; Gim, M.G.; Chung, D.K. Different immune regulatory potential of Lactobacillus plantarum and Lactobacillus sakei isolated from Kimchi. J. Microbiol. Biotechnol. 2014, 24, 1629-1635, doi:10.4014/jmb.1406.06062.

14. Won, S.M.; Chen, S.; Lee, S.Y.; Lee, K.E.; Park, K.W.; Yoon, J.H. Lactobacillus sakei ADM14 Induces Anti-Obesity Effects and Changes in Gut Microbiome in High-Fat Diet-Induced Obese Mice. Nutrients 2020, 12, doi:10.3390/nu12123703.

15. Rather, I.A.; Bajpai, V.K.; Ching, L.L.; Majumder, R.; Nam, G.J.; Indugu, N.; Singh, P.; Kumar, S.; Hajrah, N.H.; Sabir, J.S.M., et al. Effect of a bioactive product SEL001 from Lactobacillus sakei probio65 on gut microbiota and its anti-colitis effects in a TNBS-induced colitis mouse model. Saudi. J. Biol. Sci. 2020, 27, 261-270, doi:10.1016/j.sjbs.2019.09.004.

16. Rather, I.A.; Bajpai, V.K.; Huh, Y.S.; Han, Y.K.; Bhat, E.A.; Lim, J.; Paek, W.K.; Park, Y.H. Probiotic Lactobacillus sakei proBio65 Extract Ameliorates the Severity of Imiquimod Induced Psoriasis-Like Skin Inflammation in a Mouse Model. Front. Microbiol. 2018, 9, 1021, doi:10.3389/fmicb.2018.01021.

17. Jung, J.Y.; Shin, J.S.; Lee, S.G.; Rhee, Y.K.; Cho, C.W.; Hong, H.D.; Lee, K.T. Lactobacillus sakei K040706 evokes immunostimulatory effects on macrophages through TLR 2-mediated activation. Int. Immunopharmacol. 2015, 28, 88-96, doi:10.1016/j.intimp.2015.05.037.

18. Chaillou, S.; Champomier-Verges, M.C.; Cornet, M.; Crutz-Le Coq, A.M.; Dudez, A.M.; Martin, V.; Beaufils, S.; DarbonRongere, E.; Bossy, R.; Loux, V., et al. The complete genome sequence of the meat-borne lactic acid bacterium Lactobacillus sakei 23K. Nat. Biotechnol. 2005, 23, 1527-1533, doi:10.1038/nbt1160.

19. Chaillou, S.; Lucquin, I.; Najjari, A.; Zagorec, M.; Champomier-Verges, M.C. Population genetics of Lactobacillus sakei reveals three lineages with distinct evolutionary histories. PLoS One. 2013, 8, e73253, doi:10.1371/journal.pone.0073253.

20. Nyquist, O.L.; McLeod, A.; Brede, D.A.; Snipen, L.; Aakra, A.; Nes, I.F. Comparative genomics of Lactobacillus sakei with emphasis on strains from meat. Mol. Genet. Genomics. 2011, 285, 297-311, doi:10.1007/s00438-011-0608-1.

21. O' Donnell, M.M.; Harris, H.M.B.; Jeffery, I.B.; Claesson, M.J.; Younge, B.; O' Toole, P.W.; Ross, R.P. The core faecal bacterial microbiome of Irish Thoroughbred racehorses. Lett. Appl. Microbiol. 2013, 57, 492-501, doi:10.1111/lam.12137.

22. Luo, R.B.; Liu, B.H.; Xie, Y.L.; Li, Z.Y.; Huang, W.H.; Yuan, J.Y.; He, G.Z.; Chen, Y.X.; Pan, Q.; Liu, Y.J., et al. SOAPdenovo2: an empirically improved memory-efficient short-read de novo assembler. GigaScience. 2012, 1, 6, doi:10.1186/2047-217x-118.

23. Loux, V.; Coeuret, G.; Zagorec, M.; Vergès, M.-C.C.; Chaillou, S. Complete and Draft Genome Sequences of Nine Lactobacillus sakei Strains Selected from the Three Known Phylogenetic Lineages and Their Main Clonal Complexes. Genome Announc. 2018, 6, 10.1128/genomeA.00082-18.

24. Kim, K.H.; Chun, B.H.; Baek, J.H.; Roh, S.W.; Lee, S.H.; Jeon, C.O. Genomic and metabolic features of Lactobacillus sakei as revealed by its pan-genome and the metatranscriptome of kimchi fermentation. Food Microbiol. 2020, 86, 103341, doi:10.1016/j.fm.2019.103341.

25. Inglin, R.C.; Meile, L.; Stevens, M.J.A. Draft Genome Sequences of 43 Lactobacillus Strains from the Species L. curvatus, L. fermentum, L. paracasei, L. plantarum, L. rhamnosus, and L. sakei, Isolated from Food Product. Genome Announc. 2017, 5, e0063200617, doi:10.1111/j.1751-7915.2010.00215.x. 
26. Jans, C.; Lagler, S.; Lacroix, C.; Meile, L.; Stevens, M.J.A. Complete Genome Sequences of Lactobacillus curvatus KG6, L. curvatus MRS6, and Lactobacillus sakei FAM18311, Isolated from Fermented Meat Products. Genome Announc. 2017, 5, doi:10.1128/genomeA.00915-17.

27. Lim, H.I.; Lee, J.; Jang, J.Y.; Park, H.W.; Choi, H.J.; Kim, T.W.; Kang, M.R.; Lee, J.H. Draft Genome Sequence of Lactobacillus sakei Strain wikim 22, Isolated from Kimchi in Chungcheong Province, South Korea. Genome Announc. 2014, 2, doi:10.1128/genomeA.01296-14.

28. Ilıkkan, Ö.K. CRISPR/Cas systems and anti-repeat sequences of Lactobacillus curvatus, Lactobacillus graminis, Lactobacillus fuchuensis, and Lactobacillus sakei genomes. Korean Journal of Microbiology. 2021, 57, 12-22, doi:10.7845/kjm.2021.0093.

29. Kwun, S.Y.; Yoon, J.A.; Park, E.H.; Kim, M.D. Complete genome sequence data of Lactobacillus sakei MBEL1397 isolated from Kimchi. Data Brief. 2020, 31, 105740, doi:10.1016/j.dib.2020.105740.

30. Kim, J.H.; Kim, E.; Kim, C.G.; Choo, D.W.; Kim, H.Y. Draft Genome Sequence of Lactobacillus sakei Strain FBL1, a Probiotic Bacterium Isolated from Mukeunji, a Long-Fermented Kimchi, in South Korea. Genome Announc. 2016, 4, doi:10.1128/genomeA.00365-16.

31. Schuster, J.A.; Vogel, R.F.; Ehrmann, M.A. Characterization and distribution of CRISPR-Cas systems in Lactobacillus sakei. Arch. Microbiol. 2019, 201, 337-347, doi:10.1007/s00203-019-01619-x.

32. McLeod, A.; Brede, D.A.; Rud, I.; Axelsson, L. Genome Sequence of Lactobacillus sakei subsp. sakei LS25, a Commercial Starter Culture Strain for Fermented Sausage. Genome Announc. 2013, 1, doi:10.1128/genomeA.00475-13.

33. Kato, S.; Oikawa, T. Genome Sequence of Lactobacillus sakei LK-145 Isolated from a Japanese Sake Cellar as a High Producer of d-Amino Acids. Genome Announc. 2017, 5, doi:10.1128/genomeA.00656-17.

34. Mizuno, K.; Mizuno, M.; Yamauchi, M.; Takemura, A.J.; Medrano Romero, V.; Morikawa, K. Adjacent-possible ecological niche: growth of Lactobacillus species co-cultured with Escherichia coli in a synthetic minimal medium. Sci. Rep. 2017, 7, 12880, doi:10.1038/s41598-017-12894-3.

35. Fujii, T.; Yamauchi, T.; Ishiyama, M.; Gogami, Y.; Oikawa, T.; Hata, Y. Crystallographic studies of aspartate racemase from Lactobacillus sakei NBRC 15893. Acta Crystallogr. F. Struct. Biol. Commun. 2015, 71, 1012-1016, doi:10.1107/S2053230X15010572.

36. De Boeck, I.; van den Broek, M.F.L.; Allonsius, C.N.; Spacova, I.; Wittouck, S.; Martens, K.; Wuyts, S.; Cauwenberghs, E.; Jokicevic, K.; Vandenheuvel, D., et al. Lactobacilli Have a Niche in the Human Nose. Cell Rep. 2020, 31, 107674, doi:10.1016/j.celrep.2020.107674.

37. Delcher, A.L.; Bratke, K.A.; Powers, E.C.; Salzberg, S.L. Identifying bacterial genes and endosymbiont DNA with Glimmer. Bioinformatics 2007, 23, 673-679, doi:10.1093/bioinformatics/btm009.

38. Bairoch, A.; Apweiler, R. The SWISS-PROT protein sequence data bank and its supplement TrEMBL in 1999. Nucleic Acids Res. 1999, 27, 49-54, doi:10.1093/nar/27.1.49.

39. Pruitt, K.D.; Tatusova, T.; Maglott, D.R. NCBI reference sequences (RefSeq): a curated non-redundant sequence database of genomes, transcripts and proteins. Nucleic Acids Res. 2007, 35, D61-D65, doi:10.1093/nar/gk1842.

40. Zhao, Y.B.; Wu, J.Y.; Yang, J.H.; Sun, S.X.; Xiao, J.F.; Yu, J. PGAP: pan-genomes analysis pipeline. Bioinformatics 2012, 28, 416-418, doi:10.1093/bioinformatics/btr655.

41. Li, L.; Stoeckert, C.J.; Roos, D.S. OrthoMCL: Identification of ortholog groups for eukaryotic genomes. Genome Res. 2003, 13, 2178-2189, doi:10.1101/gr.1224503.

42. Valeriano, V.D.V.; Oh, J.K.; Bagon, B.B.; Kim, H.; Kang, D.K. Comparative genomic analysis of Lactobacillus mucosae LM1 identifies potential niche-specific genes and pathways for gastrointestinal adaptation. Genomics 2019, 111, 24-33, doi:10.1016/j.ygeno.2017.12.009.

43. Richter, M.; Rossello-Mora, R. Shifting the genomic gold standard for the prokaryotic species definition. Proc. Natl. Acad. Sci. USA. 2009, 106, 19126-19131, doi:10.1073/pnas.0906412106.

44. Kelleher, P.; Bottacini, F.; Mahony, J.; Kilcawley, K.N.; van Sinderen, D. Comparative and functional genomics of the Lactococcus lactis taxon; insights into evolution and niche adaptation. BMC Genomics. 2017, 18, doi:10.1186/s12864-017-3650-5.

45. Mailund, T.; Brodal, G.S.; Fagerberg, R.; Pedersen, C.N.S.; Phillips, D. Recrafting the neighbor-joining method. BMC Bioinformatics. 2006, 7, doi:10.1186/1471-2105-7-29.

46. Subramanian, B.; Gao, S.H.; Lercher, M.J.; Hu, S.N.; Chen, W.H. Evolview v3: a webserver for visualization, annotation, and management of phylogenetic trees. Nucleic Acids Res. 2019, 47, W270-W275, doi:10.1093/nar/gkz357.

47. Lombard, V.; Ramulu, H.G.; Drula, E.; Coutinho, P.M.; Henrissat, B. The carbohydrate-active enzymes database (CAZy) in 2013. Nucleic Acids Res. 2014, 42, D490-D495, doi:10.1093/nar/gkt1178.

48. Deng, W.K.; Wang, Y.B.; Liu, Z.X.; Cheng, H.; Xue, Y. HemI: A Toolkit for Illustrating Heatmaps. Plos One. 2014, 9, 5, doi:10.1371/journal.pone.0111988.

49. Jia, B.F.; Raphenya, A.R.; Alcock, B.; Waglechner, N.; Guo, P.Y.; Tsang, K.K.; Lago, B.A.; Dave, B.M.; Pereira, S.; Sharma, A.N., et al. CARD 2017: expansion and model-centric curation of the comprehensive antibiotic resistance database. Nucleic Acids Res. 2017, 45, D566-D573, doi:10.1093/nar/gkw1004.

50. Grissa, I.; Vergnaud, G.; Pourcel, C. The CRISPRdb database and tools to display CRISPRs and to generate dictionaries of spacers and repeats. BMC Bioinformatics. 2007, 8, 10, doi:10.1186/1471-2105-8-172.

51. Lorenz, R.; Bernhart, S.H.; Siederdissen, C.H.Z.; Tafer, H.; Flamm, C.; Stadler, P.F.; Hofacker, I.L. ViennaRNA Package 2.0. Algorithms. Mol. Biol. 2011, 6, 14, doi:10.1186/1748-7188-6-26. 
52. Chun, J.; Oren, A.; Ventosa, A.; Christensen, H.; Arahal, D.R.; da Costa, M.S.; Rooney, A.P.; Yi, H.; Xu, X.W.; De Meyer, S., et al. Proposed minimal standards for the use of genome data for the taxonomy of prokaryotes. Int. J. Syst. Evol. Microbiol. 2018, 68, 461-466, doi:10.1099/ijsem.0.002516.

53. Gao, Y.; Li, D.; Liu, X. Bacteriocin-producing Lactobacillus sakei C2 as starter culture in fermented sausages. Food Control. 2014, 35, 1-6, doi:10.1016/j.foodcont.2013.06.055.

54. Malheiros, P.S.; Cuccovia, I.M.; Franco, B.D.G.M. Inhibition of Listeria monocytogenes in vitro and in goat milk by liposomal nanovesicles containing bacteriocins produced by Lactobacillus sakei subsp. sakei 2a. Food Control. 2016, 63, 158-164, doi:10.1016/j.foodcont.2015.11.037.

55. Ji, Y.; Park, S.; Chung, Y.; Kim, B.; Park, H.; Huang, E.; Jeong, D.; Jung, H.Y.; Kim, B.; Hyun, C.K., et al. Amelioration of obesity-related biomarkers by Lactobacillus sakei CJLS03 in a high-fat diet-induced obese murine model. Sci. Rep. 2019, 9, 6821, doi:10.1038/s41598-019-43092-y.

56. Kim, J.Y.; Park, B.K.; Park, H.J.; Park, Y.H.; Kim, B.O.; Pyo, S. Atopic dermatitis-mitigating effects of new Lactobacillus strain, Lactobacillus sakei probio 65 isolated from Kimchi. J. Appl. Microbiol. 2013, 115, 517-526, doi:10.1111/jam.12229.

57. Seo, S.; Shin, J.-S.; Lee, W.-S.; Rhee, Y.K.; Cho, C.-W.; Hong, H.-D.; Lee, K.-T. Anti-colitis effect of Lactobacillus sakei K040706 via suppression of inflammatory responses in the dextran sulfate sodium-induced colitis mice model. J. Funct. Foods. 2017, 29, 256-268, doi:10.1016/j.jff.2016.12.045.

58. Eisenbach, L.; Geissler, A.J.; Ehrmann, M.A.; Vogel, R.F. Comparative genomics of Lactobacillus sakei supports the development of starter strain combinations. Microbiol. Res. 2019, 221, 1-9, doi:10.1016/j.micres.2019.01.001.

59. Wang, S.; Yang, B.; Ross, R.P.; Stanton, C.; Zhao, J.; Zhang, H.; Chen, W. Comparative Genomics Analysis of Lactobacillus ruminis from Different Niches. Genes (Basel). 2020, 11, doi:10.3390/genes11010070.

60. Broadbent, J.R.; Neeno-Eckwall, E.C.; Stahl, B.; Tandee, K.; Cai, H.; Morovic, W.; Horvath, P.; Heidenreich, J.; Perna, N.T.; Barrangou, R., et al. Analysis of the Lactobacillus casei supragenome and its influence in species evolution and lifestyle adaptation. BMC Genomics. 2012, 13, 18, doi:10.1186/1471-2164-13-533.

61. Jiang, J.; Yang, B.; Ross, R.P.; Stanton, C.; Zhao, J.; Zhang, H.; Chen, W. Comparative Genomics of Pediococcus pentosaceus Isolated from Different Niches Reveals Genetic Diversity in Carbohydrate Metabolism and Immune System. Front. Microbiol. 2020, 11, 253, doi:10.3389/fmicb.2020.00253.

62. Zhang, Q.; Zhang, L.; Ross, P.; Zhao, J.; Zhang, H.; Chen, W. Comparative Genomics of Lactobacillus crispatus from the Gut and Vagina Reveals Genetic Diversity and Lifestyle Adaptation. Genes (Basel). 2020, 11, doi:10.3390/genes11040360.

63. O'Riordan, N.; O'Callaghan, J.; Butto, L.F.; Kilcoyne, M.; Joshi, L.; Hickey, R.M.J.J.o.D.S. Bovine glycomacropeptide promotes the growth of Bifidobacterium longum ssp. infantis and modulates its gene expression. J. Dairy Sci.2018, 101, 6730-6741, doi:10.3168/jds.2018-14499.

64. Patryk, Z.; iley-Elkin, B.B.; Miriam, D.; Mark, B.L.; Permyakov, E.A.J.P.O. Structural and Biochemical Insights into the Peptidoglycan Hydrolase Domain of FlgJ from Salmonella typhimurium. PLoS One 2016, 11, e0149204, doi:10.1371/journal.pone.0149204.

65. Sista Kameshwar, A.K.; Qin, W. Understanding the structural and functional properties of carbohydrate esterases with a special focus on hemicellulose deacetylating acetyl xylan esterases. Mycology. 2018, 9, 273-295, doi:10.1080/21501203.2018.1492979.

66. Salminen, S.; Wright, A.v.; Morelli, L.; Marteau, P.; Brassart, D.; Vos, W.M.d.; Fonden, R.; Saxelin, M.; Collins, K.; Mogensen, G., et al. Demonstration of safety of probiotics - a review. Int. J. Food Microbiol. 1998, 44, 93-106, doi:10.1016/s01681605(98)00128-7.

67. Georgieva, R.; Yocheva, L.; Tserovska, L.; Zhelezova, G.; Stefanova, N.; Atanasova, A.; Danguleva, A.; Ivanova, G.; Karapetkov, N.; Rumyan, N., et al. Antimicrobial activity and antibiotic susceptibility of Lactobacillus and Bifidobacterium spp. intended for use as starter and probiotic cultures. Biotechnol Biotechnol Equip. 2015, 29, 84-91, doi:10.1080/13102818.2014.987450.

68. Botta, C.; Langerholc, T.; Cencic, A.; Cocolin, L. In vitro selection and characterization of new probiotic candidates from table olive microbiota. PLoS One. 2014, 9, e94457, doi:10.1371/journal.pone.0094457.

69. Roberts, M.C.; Schwarz, S. Tetracycline and Phenicol Resistance Genes and Mechanisms: Importance for Agriculture, the Environment, and Humans. J. Environ. Qual. 2016, 45, 576-592, doi:10.2134/jeq2015.04.0207.

70. Yamaguchi, A.; Ono, N.; Akasaka, T.; Noumi, T.; Sawai, T.J.J.o.B.C. Metal-tetracycline/H+ antiporter of Escherichia coli encoded by a transposon, Tn10. The role of the conserved dipeptide, Ser65-Asp66, in tetracycline transport. J. Biol. Chem. 1990, 265, doi:10.1016/S0021-9258(18)38081-5.

71. Barrangou, R.; Fremaux, C.; Deveau, H.; Richards, M.; Boyaval, P.; Moineau, S.; Romero, D.A.; Horvath, P. CRISPR provides acquired resistance against viruses in prokaryotes. Science. 2007, 315, 1709-1712, doi:10.1126/science.1138140.

72. Tyson, G.W.; Banfield, J.F. Rapidly evolving CRISPRs implicated in acquired resistance of microorganisms to viruses. Environ. Microbiol. 2008, 10, 2147483647-2147483640, doi:10.1111/j.1462-2920.2007.01444.x.

73. Mougiakos, I.; Bosma, E.F.; Vos, W.D.; Kranenburg, R.V.; John, V.J.T.i.B. Next Generation Prokaryotic Engineering: The CRISPR-Cas Toolkit. Trends Biotechnol. 2016, 575-587, doi;10.1016/j.tibtech.2016.02.004. 